\title{
Safety and Outcome after Thrombolysis in Stroke Patients with Mild Symptoms
}

\author{
M. Köhrmann ${ }^{a} \quad$ T. Nowe $^{a} \quad$ H.B. Huttner ${ }^{a} \quad$ T. Engelhorn ${ }^{b} \quad$ T. Struffert $^{b}$ \\ R. Kollmar ${ }^{a} \quad$ M. Saake ${ }^{b} \quad$ A. Doerfler ${ }^{b} \quad$ S. Schwab ${ }^{a} \quad$ P.D. Schellinger ${ }^{a}$ \\ Departments of ${ }^{\mathrm{a}}$ Neurology and ${ }^{\mathrm{b}}$ Neuroradiology, University of Erlangen, Erlangen, Germany
}

\section{Key Words}

Intravenous thrombolysis $\cdot$ Minor stroke $\cdot$ Plasminogen activator $\cdot r t-P A \cdot$ Stroke scores $\cdot$ Thrombolytic therapy

\begin{abstract}
Background: Thrombolytic therapy is frequently withheld in patients with minor stroke symptoms. However, recent studies demonstrate that a substantial proportion of these patients dies or remains permanently disabled because of underestimation of symptom severity at baseline or secondary deterioration. We aimed to assess the safety and outcome of thrombolysis therapy in patients with minor but disabling stroke symptoms. Methods: 32 patients presenting with mild symptoms were treated with intravenous recombinant tissue-type plasminogen activator between April 2006 and April 2008. Data were extracted from a prospectively collected database. Baseline demographic data, and clinical, laboratory and imaging findings were analyzed. Outcome was assessed using the modified Rankin Scale (mRS) score at 3 months and was dichotomized into favorable (mRS 0-1) versus unfavorable (mRS 2-6). Results: In the majority of patients, the left hemisphere was affected, with aphasia representing the most common symptom leading to treatment decision. The frequency of perfusion lesion $(46 \%)$ and vessel occlusion (35\%) at baseline was high but had no effect on the outcome at 3 months in our series of treated patients. Outcome was favorable in $94 \%$ of patients, and $47 \%$ recovered without any persisting symptom. Only one asymptomatic and no symptomatic hemorrhage was
\end{abstract}

\section{KARGER}

Fax +4161306 1234 E-Mail karger@karger.ch www.karger.com
(ㄷ) 2008 S. Karger AG, Basel

$1015-9770 / 09 / 0272-0160 \$ 26.00 / 0$

Accessible online at:

www.karger.com/ced observed. Conclusion: Our data support current guidelines and international licenses which give no lower National Institutes of Health Stroke Scale (NIHSS) limit for intravenous thrombolysis (IVT). Considering the accumulating evidence that the natural course in patients with mild symptoms is not as favorable as often assumed and taking the low risk of bleeding in those patients into account, patients with mild but disabling symptoms should be treated with IVT regardless of their baseline NIHSS score.

Copyright $\odot 2008$ S. Karger AG, Basel

\section{Introduction}

The proportion of patients with acute ischemic stroke receiving recombinant tissue-type plasminogen activator (rt-PA) remains low [1]. The most important reason for this is the narrow and early time window for treatment. However, even patients who would generally be eligible are often not treated because of various reasons [2]. Several studies demonstrated that a common cause for the exclusion of patients from thrombolysis is the assumption of the treating physician that symptoms are too mild to warrant a potentially dangerous therapy [2-5]. A significant proportion of those patients however deteriorates or has persistent neurological deficits that leave them permanently disabled $[2,6]$. Furthermore, a formalized neurological examination using a scoring system such as the National Institutes of Health Stroke Scale (NIHSS) may underestimate the functionally disabling 

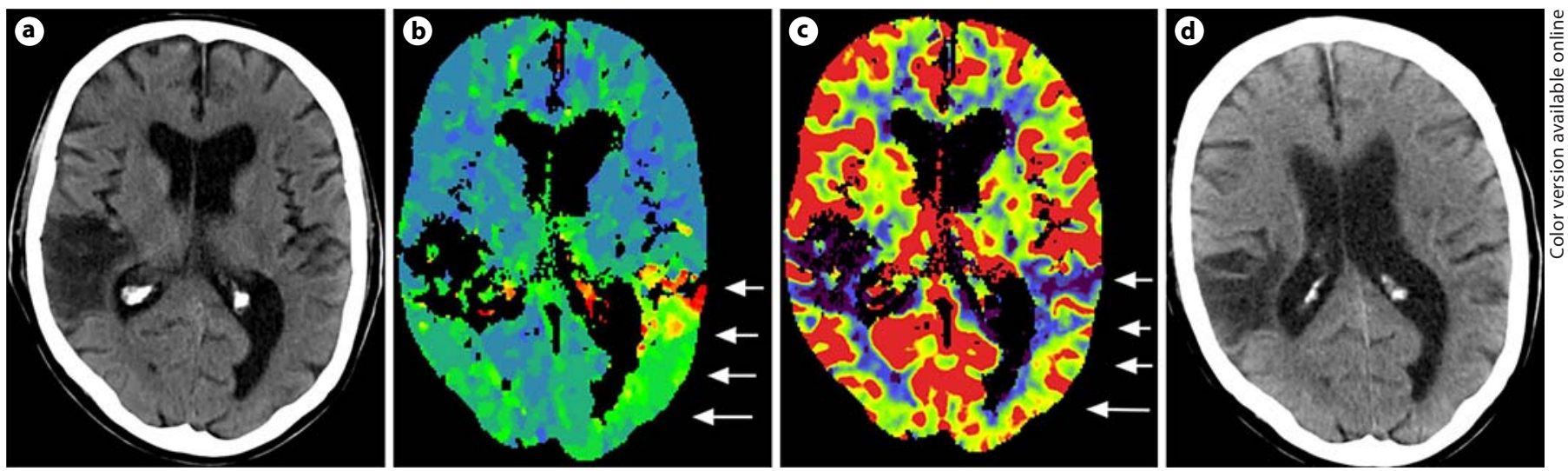

Fig. 1. Baseline and perfusion CT of a 63-year-old patient with congestive cardiomyopathy $2 \mathrm{~h}$ after onset of aphasia. a Non-contrast CT reveals only an old infarction in the right hemisphere. TTP (b) and CBV perfusion sequences (c) demonstrate hypoperfusion in the posterior part of the left MCA territory. d Follow-up CT $24 \mathrm{~h}$ after IVT reveals no new infarction.

nature of symptoms in the acute phase of stroke [7]. In the present study, we report baseline data, imaging characteristics and outcome of patients with mild strokes treated with intravenous thrombolysis (IVT).

\section{Patients and Methods}

\section{Patient Selection, Treatment and Evaluation of Outcome}

The Erlangen Stroke and Thrombolysis Database is a prospective database of all patients with acute ischemic stroke treated at our institution (University Hospital Erlangen, Germany). It was established in April 2006 and contains baseline demographic and stroke-related data as well as treatment specifics and imaging information for each patient. Data on 32 patients with mild but disabling neurological symptoms treated with IVT at our institution between April 2006 and April 2008 were retrieved from this database. There is no consensus definition of 'minor stroke'; the two most common definitions use a threshold score of $4[8,9]$ or $5[3$, $4,10]$ on the NIHSS. We chose the more severe definition of the two (NIHSS <5) to avoid underestimation of unfavorable outcomes by exclusion of patients with NIHSS scores of 4 . Patients with a pre-stroke mRS $>1$ were excluded from this analysis, as they could not achieve the endpoint of a favorable outcome (mRS $0-1$ ). According to national and international guidelines, no lower NIHSS limit was used for the selection of patients for thrombolysis $[11,12]$. Patients needed to have a disabling syndrome in the judgment of the treating stroke physician in order to be eligible for treatment $[11,12]$. Placebo-controlled randomization was ethically not justifiable because of the existing approval for the use of rt-PA in this patient group. Informed consent was obtained from the patient or next of kin in cases of off-label treatment (age/time window). All patients were treated and monitored in our stroke unit according to European guidelines [11]. NIHSS scores were recorded three times daily by a stroke neurologist certified for NIHSS evaluation. Ninety-day outcome was evaluated by a neurologist as part of the general database independently from the present study. An mRS was derived using a semistructured interview either in person or by telephone (patient/relatives). Outcome was primarily dichotomized into favorable (mRS $0-1$ ) and unfavorable (mRS 2-6), but a secondary explorative analysis was done using mRS 0 vs. 1-6.

\section{Imaging}

In addition to the standard 3-hour CT-based treatment, an MRI-mismatch-based algorithm for patients in the 3- to 9-hour time window is used at our institution as described elsewhere [13]. In brief, up to $3 \mathrm{~h}$ after stroke, patients are treated based on noncontrast CT while patients in the 3-to 9-hour window receive a multiparametric MRI protocol (DWI, PI, FLAIR, GRE, ToF and CE-MRA) as a first-line imaging modality. Between July 2006 and March 2008, our institution performed a prospective study evaluating the diagnostic power of a multiparametric CT protocol: non-contrast CT + CT angiography + CT perfusion, which was performed in all patients with suspected stroke within the 3-hour time window. The treating neurologist, however, was blinded to the results of CT angiography and CT perfusion, and patients were treated or not based on non-contrast CT only as per approval. The results of the additional imaging were made available for the present study (fig. 1). One patient was treated based on MRI findings within $3 \mathrm{~h}$ since CT was not available at the time of presentation due to another emergency. Overall 7 of the 32 patients were treated beyond $3 \mathrm{~h}$. Patients received either CT or MRI at 24-36 h to evaluate hemorrhagic complications. Asymptomatic and symptomatic hemorrhage was defined according to the criteria of the National Institute of Neurological Disorders and Stroke (NINDS) trial [14].

\section{Statistical Analysis}

Statistical analyses were performed using the Statview software package (Statview 5.0, SAS, Cary, N.C., USA; www.statview. com). All parameters are given as medians and ranges or means and SD, as appropriate. On admission, the NIHSS score was com- 
Table 1. Baseline and outcome characteristics of the patients $(\mathrm{n}=$ 32)

\begin{tabular}{lc}
\hline Age, years, median [range] & $69.5[42-92]$ \\
Male sex, $\mathrm{n}(\%)$ & $24(75)$ \\
Affected hemisphere, $\mathrm{n}(\%)$ & \\
$\quad$ Left & $21(66)$ \\
Right & $10(31)$ \\
Cerebellar & $1(3)$ \\
NIHSS score, median [range] & \\
Baseline & $3.5[1-4]$ \\
24 h & $1[0-5]$ \\
Discharge & $0[0-7]$ \\
Risk factors, $\mathrm{n}$ (\%) & \\
Hypertension & $25(78)$ \\
Hypercholesterolemia & $18(56)$ \\
Diabetes & $14(44)$ \\
Coronary heart disease & $13(41)$ \\
Atrial fibrillation & $8(25)$ \\
Previous stroke & $7(22)$ \\
Previous antiplatelet treatment, $\mathrm{n}(\%)$ & $12(38)$ \\
Temperature at baseline, ${ }^{\circ} \mathrm{C}$ & $37.1 \pm 0.5$ \\
Glucose at baseline, mg/dl & $123 \pm 51$ \\
Cholesterol at baseline, mg/dl & $204 \pm 48$ \\
TOAST criteria, $\mathrm{n}(\%)$ & \\
Large vessel & $3(9)$ \\
Embolic & $14(44)$ \\
Micro/lacunar & $12(38)$ \\
Other & $0(0)$ \\
Unknown & $3(9)$ \\
Time window, min, median [range] & $120[60-720]$ \\
Symptom leading to treatment decision, $\mathrm{n}(\%)^{1}$ & \\
Aphasia/severe dysarthria & $21(66)$ \\
Inability to ambulate & $11(34)$ \\
Paresis of the dominant hand & $10(31)$ \\
Severe neuropsychological deficits & $1(3)$ \\
Outcome at 3 months, $\mathrm{n}(\%)$ & $15(47)$ \\
mRS 0 & $15(47)$ \\
mRS 1 & $2(6)$ \\
mRS 2 & \\
\hline & \\
\hline &
\end{tabular}

${ }^{1}$ Patients can have combinations of symptoms leading to treatment decision.

pared to those after $24 \mathrm{~h}$ and at discharge using the non-parametric Wilcoxon signed rank test. A two-tailed $\mathrm{p}<0.05$ was considered statistically significant, and a Bonferroni correction for multiple testing was applied where indicated.

\section{Results}

Patient Characteristics, Clinical Course and Outcome

Patient characteristics are presented in table 1. Thirtytwo patients who received rt-PA for acute stroke with mild but disabling symptoms were identified. Median age was
69.5 years (range $42-92$ ) and $75 \%$ were male. The prevalence of cardiovascular risk factors was high. The majority of patients had left-sided infarctions reflecting the most common symptom leading to treatment which was aphasia. Other indications consisted of loss of ability to ambulate and paresis of the dominant hand often accompanied with dominant arm paresis. One patient was treated because of a severe neuropsychological syndrome consisting of inattention and confusion with an occlusion of the right M1 and only minor non-disabling motor symptoms (fig. 2).

NIHSS scores improved significantly between baseline and $24 \mathrm{~h}$ ( $\mathrm{p}<0.001$; Wilcoxon signed rank test). A trend towards an improvement after $24 \mathrm{~h}$ to discharge was still present but did not reach statistical significance (fig. 3). Only 2 patients suffered clinical deterioration between the baseline and 24-hour examinations, both patients had a day $90 \mathrm{mRS}$ score of 2 and accounted for the only unfavorable outcomes in our series. The first patient was a 70 -year-old man presenting with mild aphasia and paresis of the dominant right hand. He was treated with an NIHSS score of 3 at $2.5 \mathrm{~h}$ after symptom onset. Baseline imaging and ultrasound examination demonstrated an older left-sided carotid occlusion and a fresh distal M2-branch occlusion with a corresponding perfusion lesion. The patient worsened to NIHSS scores of 5 ( $24 \mathrm{~h}$ ) and 7 (discharge) but was stable afterwards, recovering to an mRS score of 2 at 3 months. The second patient was a 69 -year-old man with mild aphasia and severe paresis of his right dominant hand (NIHSS score of 3) treated $1.5 \mathrm{~h}$ after symptom onset. After 24 $\mathrm{h}$, the NIHSS score was 4 and although his aphasia improved to discharge (NIHSS score of 1), high-grade paresis of his right hand and an mRS score of 2 at 3 months remained despite having an NIHSS score of 0 at this time point. There was no difference in outcome between patients treated within $(n=25)$ or beyond $(n=7)$ the 3hour time window. Also, no significant correlation was found between baseline clinical or imaging parameters and outcome using a dichotomization of mRS 0 versus $1-6$ in a secondary analysis.

\section{Imaging Findings}

Imaging characteristics are shown in table 2. Patients at baseline received either non-contrast CT $(n=6)$, multiparametric CT $(\mathrm{n}=18)$ or stroke MRI $(\mathrm{n}=8)$. All patients who received CT at baseline were treated based on non-contrast CT findings. Almost half of the patients $(12 / 26 ; 46 \%)$ with perfusion imaging at baseline had a visible perfusion lesion and $36 \%$ had a visible occlusion 

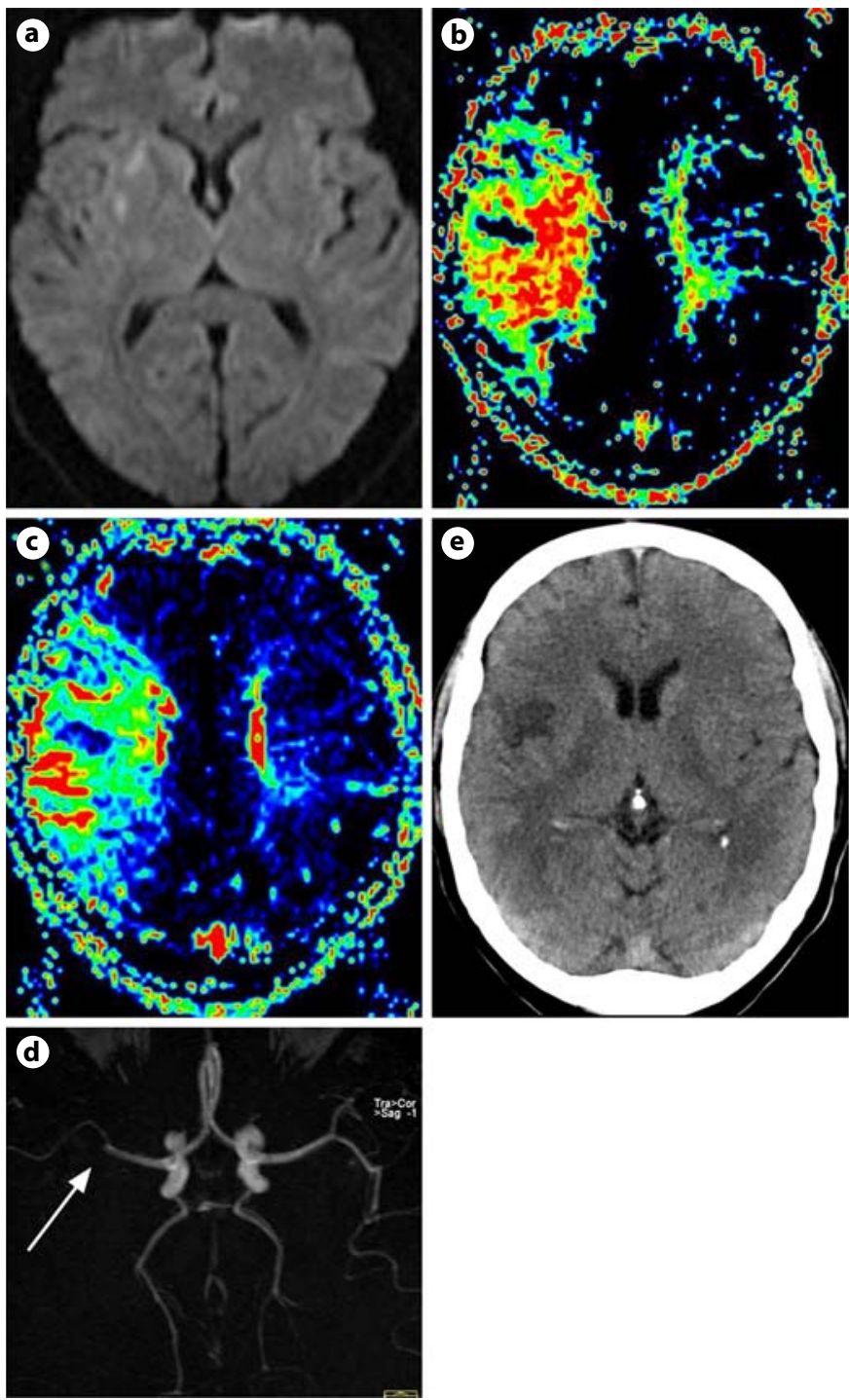

Fig. 2. MRI before treatment (a-d) and follow-up CT (e) of a 46year-old patient treated $3.5 \mathrm{~h}$ after onset of a severe neuropsychological deficit consisting of inattention and confusion with only mild left-sided motor symptoms. a DWI demonstrates two small lesions in the right insula. TTP (b) and CBV (c) perfusion maps with a large lesion corresponding to an M1-M2 occlusion demonstrated on ToF-MR angiography (d, arrow). e Follow-up CT after $24 \mathrm{~h}$ reveals only a small infarction in the right insular region.

on CT/MR angiography. Perfusion lesions were more prevalent in the MRI group (100 vs. $22 \%$; p < 0.001), reflecting a selection bias since MRI was used as a first-line imaging modality in the expanded time window $>3 \mathrm{~h}$ and patients were only treated based on PI/ DWI mismatch. In 20 of the 32 patients (63\%) and in all patients with MRI $(n=8)$ at follow-up, infarction was

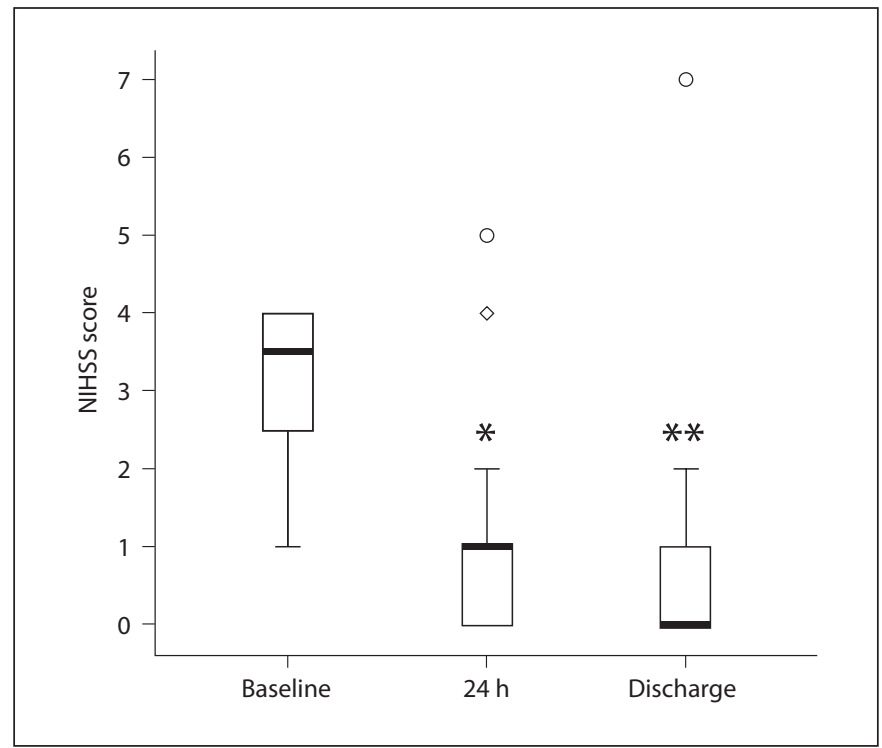

Fig. 3. Box plot of NIHSS scores at baseline, $24 \mathrm{~h}$ and at discharge (median, interquartile range and range). ${ }^{*} \mathrm{p}<0.001$ : 24 -hour scores are significantly improved compared to baseline (Wilcoxon signed rank test using Bonferroni correction); ${ }^{* *} \mathrm{p}=0.1$ : discharge scores are significantly improved compared to baseline but not compared to 24-hour scores (Wilcoxon signed rank test using Bonferroni correction).

proven by imaging. Among the 2 patients with an unfavorable outcome, 1 patient had a perfusion lesion with proven vessel occlusion (internal carotid artery and M2) whereas the other had none. Both patients had visible infarctions on follow-up imaging. The imaging profile (perfusion lesion, vessel occlusion or infarct imaging positive/negative) did not influence outcome neither when dichotomized into mRS scores 0 versus $1-6$ nor $0-1$ versus $2-6$.

There was one asymptomatic intracranial hemorrhage (ICH) which was seen in an 83-year-old patient who was treated $2 \mathrm{~h}$ after onset of a right-sided, distally pronounced arm paresis. The patient had fully recovered on day 1 and no infarction was found apart from the asymptomatic $\mathrm{ICH}$ (fig. 4).

\section{Discussion}

We assessed safety and outcome in acute stroke patients who received IVT with rt-PA and had an NIHSS score at baseline $\leq 4$. In general, these stroke patients are considered to have a 'mild' or a 'minor' stroke, and, there- 
Table 2. Neuroradiological findings

\begin{tabular}{lr}
\hline Baseline imaging modality & $\mathrm{n}(\%)$ \\
\hline Non-contrast CT & $6(19)$ \\
$\quad$ Multiparametric stroke CT & $18(56)$ \\
$\quad$ Multiparametric stroke MRI & $8(25)$ \\
Baseline imaging characteristics & $12 / 26(46)$ \\
$\quad$ Perfusion deficit (CT/MR perfusion) & $9 / 26(35)$ \\
$\quad$ Vessel occlusion (CT/MR angiography) & $12 / 24(50)$ \\
Infarct on follow-up imaging & $8 / 8(100)$ \\
$\quad$ Patients with follow-up CT & $20 / 32(63)$ \\
Patients with follow-up MRI & $1 / 32(3)$ \\
Patients with imaging-proven infarction & $0 / 32(0)$ \\
Hemorrhagic complications ${ }^{2}$ & \\
Asymptomatic ICH & \\
Symptomatic ICH & \\
\hline & 1 Only non-contrast CT was used for treatment decision. \\
2 &
\end{tabular}

fore, treatment with rt-PA is frequently omitted. In our series consisting of 32 patients, no symptomatic hemorrhages occurred and 30 of 32 patients experienced a favorable outcome (mRS score 0 or 1 ). Should these patients have been treated or would the outcome have been the same if thrombolytic therapy had been withheld? The presumed existence of any established lower or upper threshold on the NIHSS for (or against) thrombolytic therapy is a widely existing misperception even among stroke neurologists. However, neither national or international guidelines nor European or FDA licenses impose such a limit $[11,12]$. The FDA label is based on the approval-relevant NINDS study and states that 'treatment in patients with only minor symptoms was not evaluated and is therefore not recommended' (US License 1048). Nevertheless, to be eligible for inclusion into the NINDS study, patients only needed to have an acute neurological syndrome with a measurable deficit on the NIHSS [14]. Thus even patients with an NIHSS score of 1 were enrolled. In fact, none of the other large randomized controlled trials on IVT testing the 3-hour time window applied a lower NIHSS limit but usually used the phrasing that patients with 'minor symptoms' should not be enrolled. In both European Cooperative Acute Stroke Studies, patients needed to have a Scandinavian Stroke Scale score $<50$, however, no limit was set for the NIHSS score $[15,16]$. These vague exclusion criteria have led to a lot of confusion and a multitude of inconsistent definitions of 'minor stroke'.
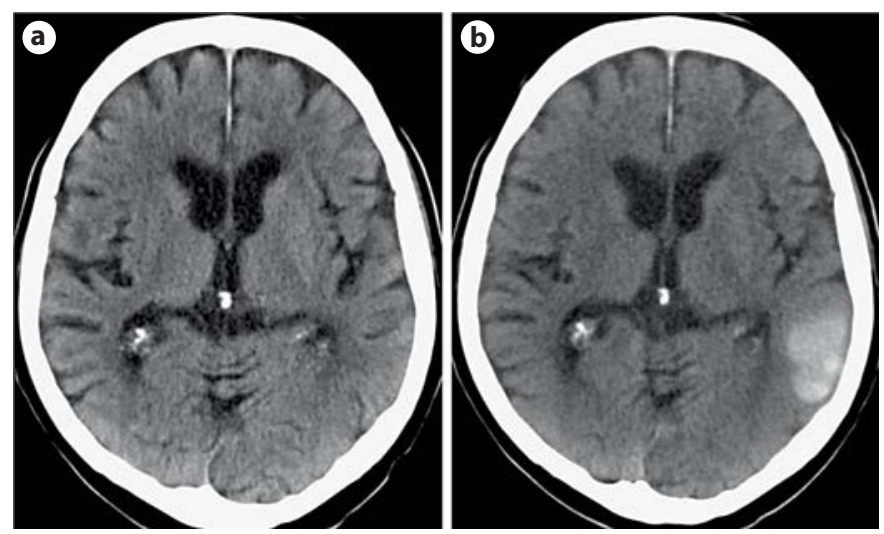

Fig. 4. Non-contrast CT at baseline (a) and after $24 \mathrm{~h}$ (b) of an 83year-old patient treated at $2 \mathrm{~h}$ for a mild right-sided hemiparesis with high-grade paresis of the dominant right hand. The patient was completely asymptomatic on day 1 , but CT shows a small cortical ICH (b).

What is a minor stroke? A post hoc analysis of the NINDS study used five different definitions for minor stroke, all of which included patients with an NIHSS score of up to 9 points, some of them even higher [17]. In some reports dealing with reasons for the exclusion of patients from IVT, patients with an NIHSS score $<5$ were generally considered ineligible for treatment $[3,4,10]$. Again, others used an NIHSS score $<4$ as a cutoff $[8,9$, 18]. Despite the complete lack of a consensus regarding the definition of 'mild stroke' symptoms or a 'minor stroke', the latter was and still is one of the most frequent reasons why IVT is withheld in patients otherwise eligible for thrombolytic therapy [2-5]. The number of patients excluded from IVT because of minor stroke within the 3-hour time window range from around 15 up to $43 \%$ in population-based analyses [2-5].

The main reason for exclusion of minor stroke patients is the assumption that those patients will have a favorable outcome with or without treatment and that it is therefore not justifiable to subject them to a potential risk for complications. However, there is accumulating evidence that the natural course of those patients is not as benign as often assumed. In most series of untreated patients with minor stroke, almost one third of patients remains permanently disabled, cannot be discharged home or die during hospitalization or within the first 3 months. This might be explained by the fact that a substantial number of patients have pathological findings in advanced imaging studies despite their 'mild' stroke symptoms, as experienced in our series. Almost half of the patients had ei- 
ther a perfusion deficit or a vessel occlusion, or both. Because this information was used for treatment decision in only $25 \%$ of patients (selection bias in the MRI group), the prevalence is still substantial even in non-selected patients with minor stroke. This finding emphasizes the risk for deterioration or lack of improvement in patients with minor stroke. Of course, with only 2 patients with an unfavorable outcome we could not identify risk factors for a poor outcome. However, other comparable studies using multiparametric imaging indicate that patients with persisting proximal vessel occlusion might be at a higher risk for early clinical deterioration and a poor functional outcome $[9,10]$.

We used an algorithm in which patients with mild stroke symptoms are treated once a disabling syndrome is present independently of the NIHSS score. In this context, it is important to perform simple functional tests in the emergency room in addition to a standardized neurological examination, such as the NIHSS, to allow the detection of disabling deficits in communication skills and usage of the dominant hand, for example. In our series, none of the $32 \mathrm{rt}-\mathrm{PA}$-treated patients remained permanently dependent or died during the 3-month followup. Of course our data cannot be used as a proof of efficacy since no control group was available, and a comparison to historical controls is methodologically invalid. The early and statistically significant improvement after IVT observed in our series is however untypical for a natural course even after stroke with mild symptoms. This has been illustrated recently regarding reperfusion time patterns in IVT-treated and untreated patients [19]. The 2 patients with a final mRS of 2 on day 90 were the only patients with a deterioration in the NIHSS score between baseline and $24 \mathrm{~h}$. There are only a few reports on IVT in patients with minor stroke, which were mainly derived from post hoc analyses of the NINDS study data. Ingall et al. [20] found insufficient evidence to declare different treatment effects throughout 5 NIHSS quintiles. One has to notice though that especially in the placebo group numbers of patients in the least severe quintile (NIHSS scores $0-5$ ) were low (16 placebo vs. $42 \mathrm{rt}-\mathrm{PA}$ patients). The same conclusion was drawn in the pooled analysis by Hacke et al. [21], which found 'no strong evidence to support exclusion of patients from treatment based on their initial NIHSS scores'. In another post hoc analysis of the NINDS study, a positive treatment effect was found throughout all five definitions for minor stroke [17].

A further, frequently encountered criticism with regard to IVT treatment of minor strokes is that in fact transient ischemic attacks were treated. This cannot be disproven without a control group. Taking into account that $63 \%$ of patients - and all patients undergoing MRI as a follow-up imaging modality - had proof of infarction and considering the high prevalence of vascular or perfusion lesions at baseline, it appears highly unlikely that giving IVT to patients with minor stroke is in fact treatment of transient ischemic attacks. We did not find any correlation between initial perfusion lesions or vessel occlusion and outcome in our cohort. However, all of our patients received IVT, so the lack of correlation can be at least partially explained by a thrombolysis-associated recanalization and reperfusion rate. Since no systematic examination of reperfusion was performed in our cohort, we are unable to prove this hypothesis.

In addition, the assumption that patients with minor stroke will have a favorable outcome without specific treatment is only one aspect for withholding IVT. Treating physicians weigh a presumed small potential benefit against the fear of hemorrhagic complications. In all large trials and post hoc analyses, the NIHSS score at baseline is the single most important risk factor and predictor for symptomatic ICH besides age $[22,23]$. Therefore, treatment of minor stroke patients appears to be considerably safer than in a standard unselected stroke population. This is also reflected in the bleeding rates of our series with one (possibly treatment-associated) asymptomatic but no symptomatic ICH.

Besides the above-mentioned issues, the most notable limitation of our study is the uncontrolled, non-randomized design, which therefore does not allow to draw firm conclusions regarding the efficacy of IVT in minor stroke patients. However, a placebo-controlled trial is not feasible since - at least under the present regulations - there is a clear indication for thrombolysis in disabling stroke syndromes regardless of the baseline NIHSS score, and thus patients cannot be randomized for ethical reasons.

To our knowledge, we report the first but small series of thrombolysed patients with 'minor' stroke apart from the above-mentioned post hoc analysis of the NINDS study, which itself included only a limited number of patients in its respective subgroup. Another strength of our series is that data including outcomes were prospectively collected in a database independently of the present study.

In conclusion we would like to point out that neither national/international guidelines nor official licensing justify the exclusion of patients with 'minor strokes' solely based on a specific NIHSS score. Considering the accumulating evidence that the natural course in these pa- 
tients is not as favorable as often assumed, special attention should be given to the evaluation of a disabling character of presenting symptoms. Advanced imaging techniques might help to select patients at a higher risk for clinical deterioration and unfavorable outcome in the future. At present, in concordance with present guidelines, patients with minor but disabling symptoms should be treated with IVT.

\section{Conflicts of Interest}

M.K., P.D.S. and S.S. received travel grants from Boehringer Ingelheim, and P.D.S. and S.S. are members of the speaker's bureau of Boehringer Ingelheim, the manufacturer of rt-PA (alteplase).

\section{References}

1 Reed SD, Cramer SC, Blough DK, Meyer K, Jarvik JG: Treatment with tissue plasminogen activator and inpatient mortality rates for patients with ischemic stroke treated in community hospitals. Stroke 2001;32:18321840 .

2 Barber PA, Zhang J, Demchuk AM, Hill MD, Buchan AM: Why are stroke patients excluded from TPA therapy? An analysis of patient eligibility. Neurology 2001;56:1015-1020.

3 Cocho D, Belvis R, Marti-Fabregas J, Molina-Porcel L, Diaz-Manera J, Aleu A, Pagonabarraga J, Garcia-Bargo D, Mauri A, MartiVilalta JL: Reasons for exclusion from thrombolytic therapy following acute ischemic stroke. Neurology 2005;64:719-720.

4 Kleindorfer D, Kissela B, Schneider A, Woo D, Khoury J, Miller R, Alwell K, Gebel J, Szaflarski J, Pancioli A, Jauch E, Moomaw C, Shukla R, Broderick JP: Eligibility for recombinant tissue plasminogen activator in acute ischemic stroke: a population-based study. Stroke 2004;35:e27-e29.

5 O'Connor RE, McGraw P, Edelsohn L: Thrombolytic therapy for acute ischemic stroke: why the majority of patients remain ineligible for treatment. Ann Emerg Med 1999;33:9-14

6 Smith EE, Abdullah AR, Petkovska I, Rosenthal E, Koroshetz WJ, Schwamm LH: Poor outcomes in patients who do not receive intravenous tissue plasminogen activator because of mild or improving ischemic stroke. Stroke 2005;36:2497-2499.

7 van Swieten JC, Koudstaal PJ, Visser MC, Schouten HJ, van Gijn J: Interobserver agreement for the assessment of handicap in stroke patients. Stroke 1988;19:604-607.

8 Katzan IL, Hammer MD, Hixson ED, Furlan AJ, Abou-Chebl A, Nadzam DM: Utilization of intravenous tissue plasminogen activator for acute ischemic stroke. Arch Neurol 2004; 61:346-350.

9 Nedeltchev K, Schwegler B, Haefeli T, Brekenfeld C, Gralla J, Fischer U, Arnold M, Remonda L, Schroth G, Mattle HP: Outcome of stroke with mild or rapidly improving symptoms. Stroke 2007;38:2531-2535.
10 Rajajee V, Kidwell C, Starkman S, Ovbiagele B, Alger JR, Villablanca P, Vinuela F, Duckwiler G, Jahan R, Fredieu A, Suzuki S, Saver JL: Early MRI and outcomes of untreated patients with mild or improving ischemic stroke. Neurology 2006;67:980-984.

11 European Stroke Organisation (ESO) Executive Committee, ESO Writing Committee: Guidelines for management of ischaemic stroke and transient ischaemic attack 2008. Cerebrovasc Dis 2008;25:457-507.

12 Adams HP Jr, del Zoppo G, Alberts MJ, Bhat DL, Brass L, Furlan A, Grubb RL, Higashida RT, Jauch EC, Kidwell C, Lyden PD, Morgenstern LB, Qureshi AI, Rosenwasser RH, Scott PA, Wijdicks EF: Guidelines for the early management of adults with ischemic stroke: a guideline from the American Heart Association/American Stroke Association Stroke Council, Clinical Cardiology Council, Cardiovascular Radiology and Intervention Council, and the Atherosclerotic Peripheral Vascular Disease and Quality of Care Outcomes in Research Interdisciplinary Working Groups: the American Academy of Neurology affirms the value of this guideline as an educational tool for neurologists. Stroke 2007;38:1655-1711.

13 Köhrmann M, Juttler E, Huttner HB, Nowe T, Schellinger PD: Acute stroke imaging for thrombolytic therapy - an update. Cerebrovasc Dis 2007;24:161-169.

14 Tissue plasminogen activator for acute ischemic stroke. The National Institute Of Neurological Disorders and Stroke rt-PA Stroke Study Group. N Engl J Med 1995;333:15811587.

15 Hacke W, Kaste M, Fieschi C, Toni D, Lesaffre E, von Kummer R, Boysen G, Bluhmki E, Hoxter G, Mahagne $\mathrm{MH}$, et al: Intravenous thrombolysis with recombinant tissue plasminogen activator for acute hemispheric stroke. The European Cooperative Acute Stroke Study (ECASS). JAMA 1995;274: 1017-1025.

16 Hacke W, Kaste M, Fieschi C, von Kummer R, Davalos A, Meier D, Larrue V, Bluhmki E, Davis S, Donnan G, Schneider D, Diez-Tejedor E, Trouillas P: Randomised double-blind placebo-controlled trial of thrombolytic therapy with intravenous alteplase in acute ischaemic stroke (ECASS II). Second European-Australasian Acute Stroke Study Investigators. Lancet 1998;352:1245-1251.
17 Recombinant tissue plasminogen activator for minor strokes: The National Institute of Neurological Disorders and Stroke rt-PA Stroke Study experience. Ann Emerg Med 2005;46:243-252.

18 Clark WM, Wissman S, Albers GW, Jhamandas JH, Madden KP, Hamilton S: Recombinant tissue-type plasminogen activator (alteplase) for ischemic stroke 3 to 5 hours after symptom onset. The ATLANTIS study: a randomized controlled trial. Alteplase Thrombolysis for Acute Noninterventional Therapy in Ischemic Stroke. JAMA 1999; 282:2019-2026.

19 Merino JG, Latour LL, An L, Hsia AW, Kang DW, Warach S: Reperfusion half-life: a novel pharmacodynamic measure of thrombolytic activity. Stroke 2008;39:2148-2150.

20 Ingall TJ, O'Fallon WM, Asplund K, Goldfrank LR, Hertzberg VS, Louis TA, Christianson TJ: Findings from the reanalysis of the NINDS tissue plasminogen activator for acute ischemic stroke treatment trial. Stroke 2004;35:2418-2424.

21 Hacke W, Donnan G, Fieschi C, Kaste M, von Kummer R, Broderick JP, Brott T, Frankel M, Grotta JC, Haley EC Jr, Kwiatkowski T, Levine SR, Lewandowski C, Lu M, Lyden P, Marler JR, Patel S, Tilley BC, Albers G, Bluhmki E, Wilhelm M, Hamilton S: Association of outcome with early stroke treatment: pooled analysis of ATLANTIS, ECASS, and NINDS rt-PA stroke trials. Lancet 2004; 363:768-774.

22 Köhrmann M, Juttler E, Fiebach JB, Huttner $\mathrm{HB}$, Siebert S, Schwark C, Ringleb PA, Schellinger PD, Hacke W: MRI versus CTbased thrombolysis treatment within and beyond the $3 \mathrm{~h}$ time window after stroke onset: a cohort study. Lancet Neurol 2006;5: 661-667.

23 Schellinger PD, Thomalla G, Fiehler J, Kohrmann M, Molina CA, Neumann-Haefelin T, Ribo M, Singer OC, Zaro-Weber O, Sobesky J: MRI-based and CT-based thrombolytic therapy in acute stroke within and beyond established time windows: an analysis of 1,210 patients. Stroke 2007;38:2640-2645. 